\title{
D(d,p)t REACTION RATE ENHANCEMENT IN A MIXED LAYER OF Au AND Pd
}

\author{
A. KITAMURA, Y. AWA, T. MINARI, N. KUBOTA, A. TANIIKE and Y. FURUYAMA \\ Department of Nuclear Engineering, Kobe University of Mercantile Marine \\ 5-1-1 Fukaeminami-machi, Higashinada-ku, Kobe 658-0022, Japan \\ E-mail:kitamura@cc.kshosen.ac.jp
}

\begin{abstract}
To investigate possible anomalies in nuclear reactions in solids, deuterium ion irradiation of deuterated $\mathrm{Au} / \mathrm{Pd}$ samples have been performed with extensive measurements of reaction products and in situ characterization of the samples including ERDA and RBS. The $\mathrm{D}(\mathrm{d}, \mathrm{p}) \mathrm{t}$ reaction rate has been observed to become three orders of magnitude greater than the calculated one. The deuterium density distribution in the sample with a composition modified by irradiation has been observed to peak at a depth appreciably greater than the projectile range. We speculate that the formation of the mixed layer of $\mathrm{Au}$ and Pd maintaining significantly high deuterium density is the key factor for the reaction rate enhancement.
\end{abstract}

\section{Introduction}

A variety of deuterium-induced reaction in metal deuterides with some yield anomaly has been reported in recent years. Three-body reactions, i.e., $\mathrm{DD}\left(\mathrm{d},{ }^{4} \mathrm{He}\right) \mathrm{pn}, \mathrm{DD}\left(\mathrm{d},{ }^{4} \mathrm{He}\right) \mathrm{d}$ and $\mathrm{DD}\left(\mathrm{d},{ }^{3} \mathrm{He}\right) \mathrm{t}$, have been claimed to take place with great enhancements of the reaction rate in $\mathrm{TiD}_{\mathrm{x}}$ samples bombarded with deuterons in the $100 \mathrm{keV}$ energy region [1-3]. The $\mathrm{D}(\mathrm{d}, \mathrm{p}) \mathrm{t}$ reaction rate also has been measured in the energy region of several $\mathrm{keV}$ in various samples [4] to find that the nuclear reaction rate could be enhanced in a condensed matter. At $E_{\mathrm{d}}=2.5 \mathrm{keV}$, it is enhanced by a factor of fifty from the bare deuteron rate and the screening energy deduced from the excitation function amounts to $600 \mathrm{eV}$.

Moreover, nuclear transmutations in a variety of electrodes subjected to electrolysis or discharges have been reported by many authors, which includes experiments on forced permeation of $\mathrm{D}$ through a multi-layered film of $\mathrm{Pd}$ and $\mathrm{CaO}[5,6]$.

In these reports, the deuterium densities in the samples have not always been made clear during these events. The three-body reaction probabilities were measured with the titanium deuterides. However, the deuterium density under irradiation was assumed. In some experiments of electrolysis and nuclear transmutation, the deuterium densities were measured by resistivity of the sample, which gives values averaged over the sample. Measurements of the local deuterium density in samples should be effective [7] to identify the anomaly and clarify the origin of anomalous enhancements of the reaction rate.

In the present paper, we report experimental results on the $\mathrm{D}(\mathrm{d}, \mathrm{p}) \mathrm{t}$ reaction rate and deuterium density measured in situ and simultaneously by accelerator analyses under 
deuterium irradiation of gold-deposited palladium $(\mathrm{Au} / \mathrm{Pd})$ samples. The experimental system is also capable of in situ loading of the target sample with deuterium [8].

\section{Experimental Procedure}

\subsection{Experimental apparatus}

A schematic of the experimental system is shown in Fig. 1. A 30-kV duoplasmatron ion source followed by a Wien filter supplies atomic/molecular deuterium ion beams that are used as the irradiation beam to induce nuclear events in the sample target located at the center of the target chamber. The beams bombard the front surface of the sample in vacuum whose rear surface is exposed to deuterium gas in a reservoir at a pressure of 0.1 MPa typically. The current and the ion energy range between $1-30 \mu \mathrm{A}$ and $7.5-27$ $\mathrm{keV}$, respectively, in the form of $\mathrm{D}^{+}, \mathrm{D}_{2}{ }^{+}$and $\mathrm{D}_{3}{ }^{+}$ions. The $\Delta \mathrm{E}$-E counter telescope technique is employed to measure the mass and energy of the $\mathrm{D}(\mathrm{d}, \mathrm{p}) \mathrm{t}$ reaction products simultaneously. A $20-\mu \mathrm{m}$-thick totally depleted solid state detector (SSD) followed by a partially depleted SSD with a 500- $\mu$ m-thick depletion layer is located at an angle of $145^{\circ}$ with respect to the beam direction.

In the present experiments, 1-mm-thick Pd plates have been prepared as the sample target. The Pd has a high permeability for hydrogen and the hydrogen transport is known to be recombination limited rather than diffusion limited. Most of them were therefore coated with a Au layer deposited by sputtering evaporation. The Au layer is expected to keep the deuterium density high in the Pd bulk. While Au has a lattice constant very close to that of $\mathrm{Pd}$, the saturation density of deuterium in $\mathrm{Au}$ is very low $\left(\mathrm{AuD}_{0.036}\right)$. The thickness of the Au layer ranges from 20 to $140 \mathrm{~nm}$ as measured with ${ }^{4} \mathrm{He}-\mathrm{RBS}$ method.

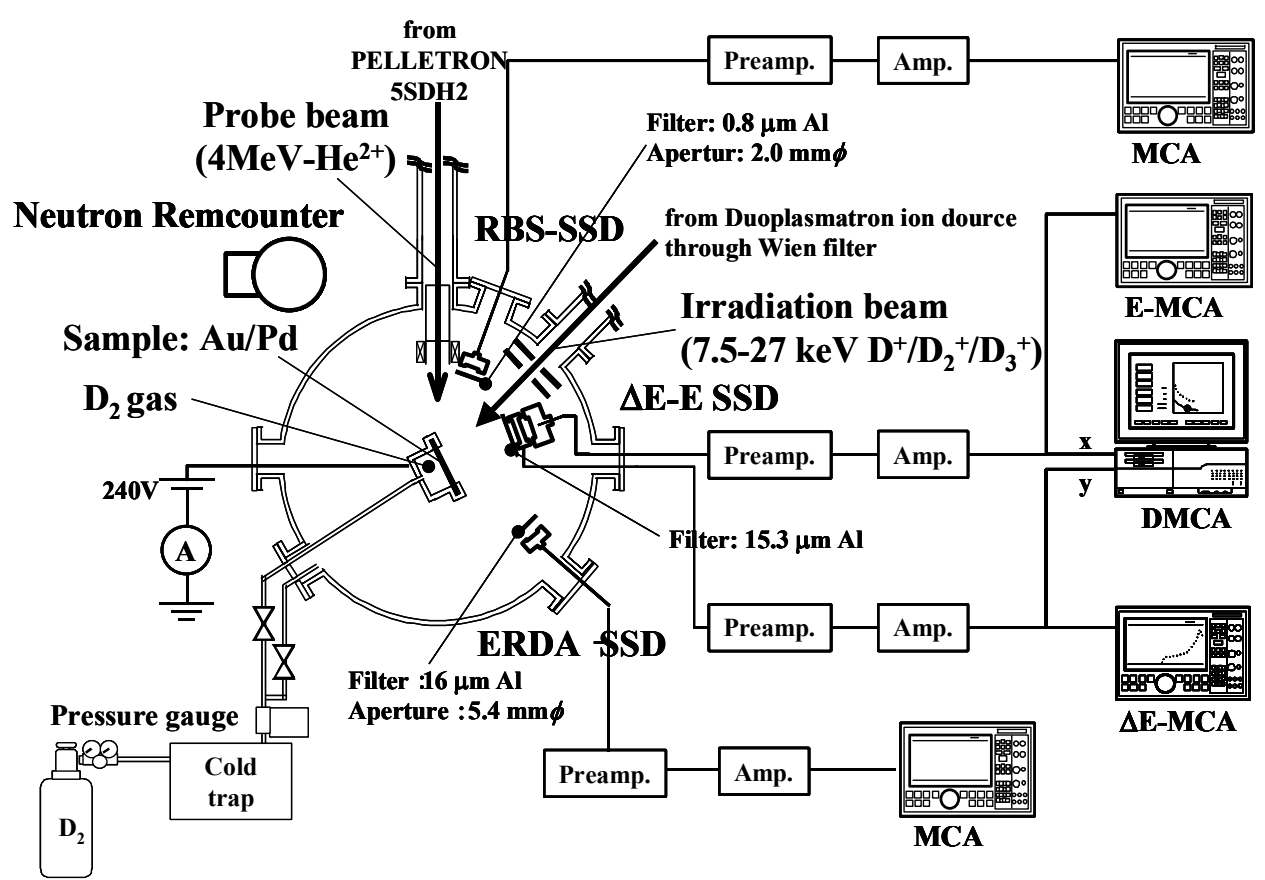

Fig.1. Schematic of the low-energy ion implantation system capable of in situ absorption of gas and accelerator analyses. 
The sample can be characterized in situ and simultaneously by means of accelerator analyses, i.e. Elastic Recoil Detection Analysis (ERDA), and Rutherford Backscattering Spectroscopy (RBS), during/after irradiation. The Pelletron 5SDH2 supplies 1- $10 \mathrm{nA}$ beams of $4 \mathrm{MeV}^{4} \mathrm{He}^{2+}$ as probing ions. Two SSDs are prepared around the sample to detect scattered and recoil particles for RBS at $160^{\circ}$ and for ERDA at $34^{\circ}$, respectively, with respect to the analyzing accelerator beam. Thin aluminum films are mounted on all SSDs to shield them from thermal radiation and low-energy secondary particles emitted from the target heated by the beam irradiation. The incident probe beam dose for every analysis is monitored by the spectral height of the bulk Pd in the RBS spectrum itself.

\subsection{Data analysis}

The $\mathrm{D}(\mathrm{d}, \mathrm{p}) \mathrm{t}$ reaction rate in the sample is evaluated from the proton yield measured with the $\Delta \mathrm{E}-\mathrm{E}$ detector. An example of the spectrum recorded on the E-SSD is shown in Fig.2, where the $\mathrm{D}(\mathrm{d}, \mathrm{p}) \mathrm{t}$ protons are identified at an energy of $2.4 \mathrm{MeV}$. The reaction probability $R_{\mathrm{m}}$ is calculated from the measured proton yield $Y_{\text {ROI }}$ during bombardment with $N_{\mathrm{i}}$ incident deuterons;

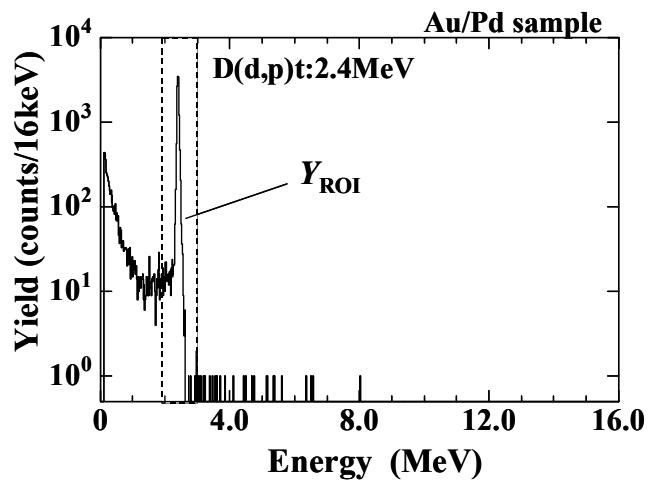

Fig.2. Typical energy spectrum observed on E-SSD.

where $\Delta \Omega$ is the solid angle of the detector.

We introduce an enhancement factor for the $\mathrm{D}(\mathrm{d}, \mathrm{p}) \mathrm{t}$ reaction probability, $R_{\mathrm{r}}$, in order to evaluate the difference between the measured reaction rate and that predicted by the conventional theory. The $R_{\mathrm{r}}$ is defined as the ratio of the measured probability $R_{\mathrm{m}}$ to the calculated one $R_{\mathrm{c}}$;

$$
R_{\mathrm{r}} \equiv \frac{R_{\mathrm{m}}}{R_{\mathrm{c}}}
$$

To calculate the $\mathrm{D}(\mathrm{d}, \mathrm{p}) \mathrm{t}$ reaction probability $R_{\mathrm{c}}$ for a given composition of target atoms and thickness, either Monte Carlo method or continuous slowing down model (CSD) is used. In the former, the probability $R_{\mathrm{c}}$ is calculated to be an averaged sum of the probabilities that each collision with $\mathrm{D}$ in the substrate results in the nuclear reaction;

$$
R_{\mathrm{c}}=\overline{\sum_{i} \frac{\sigma_{\mathrm{s}}\left(E_{i}\right)}{\pi b_{\max , i}^{2}}},
$$

where $\sigma\left(E_{\mathrm{i}}\right)$ is the nuclear reaction cross section at a colliding energy of $E_{\mathrm{i}}$, and $b_{\max }$ is the maximum impact parameter. Modification to include eq.(3) in the Monte Carlo routine has been made both to the TRIM code [9] (TRIM85N [7]) and the TRIDYN code [10] (TRINDYN). In the CSD the following expression is used: 


$$
R_{\mathrm{c}}=\int_{E_{\mathrm{in}}}^{0} \frac{n_{\mathrm{D}} \sigma_{\mathrm{s}}\left(E_{\mathrm{c}}\right)}{(\mathrm{d} E / \mathrm{d} x)} \mathrm{d} E
$$

where $n_{\mathrm{D}}$ is the deuterium density in the target, $-\mathrm{d} E / \mathrm{d} x$ the stopping power of the target, $E_{\mathrm{c}}$ the energy in the center-of-mass system of the reacting deuteron pair, and $E_{\text {in }}$ is the incident energy of the projectile deuteron.

The reaction cross section $\sigma_{\mathrm{s}}(E)$ involves a barrier penetration probability $P_{\mathrm{s}}(E)$. In the present case of reactions in solids, a screened Coulomb potential $V_{\mathrm{s}}(r)$ (universal potential [9]) is used;

$$
\begin{aligned}
& \sigma_{\mathrm{s}}(E)=\frac{S(E)}{E} P_{\mathrm{s}}(E)=\frac{S(E)}{E} \exp \left[-\int_{r_{\mathrm{n}}}^{r_{\mathrm{c}}} \frac{\sqrt{2 M\left(V_{\mathrm{s}}(r)-E\right)}}{\hbar} \cdot \mathrm{d} r\right], \\
& V_{\mathrm{s}}(r)=\frac{z_{1} z_{2} e^{2}}{r} \Phi\left(\frac{r}{r_{\mathrm{U}}}\right),
\end{aligned}
$$

where $\Phi\left(r / r_{\mathrm{U}}\right)$ is the interatomic screening function, $r_{\mathrm{U}}$ is the interatomic screening distance [9], and $S(E)$ is the astrophysical S-factor expressed as the empirical formula [11]. Other notations used are traditional ones.

Some examples of the calculated $R_{\mathrm{c}}$ are shown in Table 1 for $7.5 \mathrm{keV}$ deuteron incidence. The saturation composition of $\mathrm{AuD}_{0.036}$ and $\mathrm{PdD}_{0.86}$ is assumed in the calculation with CSD and TRIM85N. Agreement between the results with CSD and TRIM85N is within a factor of two. However, TRINDYN gives a rather large value compared with others. Moreover, it can hardly be adopted to the cases of the $\mathrm{Au}$ deposited targets, since we do not know the saturation density of deuterium, which the code requires as one of the input parameters, in the material with arbitrary mixing ratio of $\mathrm{Au}$ and Pd. In view of the above differences, we should admit uncertainty of a factor of about three in the values of $R_{\mathrm{c}}$.

The $R_{\mathrm{c}}$ is reduced by more than one order of magnitude when the target surface is covered with a D-saturated Au layer with thickness comparable to the deuteron range. In the following discussion of the enhancement factor $R_{\mathrm{r}}$, we assume a uniform saturation composition of $\mathrm{PdD}_{0.86}$ all over the projectile range; the $R_{\mathrm{c}}$ for $\mathrm{PdD}_{0.86}$ is used in eq.(3) regardless of existence of the Au atoms on the target surface. It should therefore be noted that the enhancement factor $R_{\mathrm{r}}$ derived in the following will be the minimum values. If the TRINDYN result is used for $R_{\mathrm{c}}$, however, the $R_{\mathrm{r}}$ would be smaller by a factor of about three.

Table 1. Reaction probabilities calculated with the continuous slowing down model (CSD), TRIM85N and TRINDYN for various targets irradiated with $7.5 \mathrm{keV} \mathrm{D}^{+}$.

\begin{tabular}{|c|c|c|c|}
\hline \multirow{2}{*}{ Target composition } & \multicolumn{3}{|c|}{ Reaction probability, $\boldsymbol{R}_{\mathrm{c}}$ (/deuteron) } \\
\cline { 2 - 4 } & CSD & TRIM85N & TRINDYN \\
\hline PdD $_{0.86}$ & $9.1 \mathrm{E}-14$ & $7.8 \mathrm{E}-14$ & $1.9 \mathrm{E}-13$ \\
\hline $\mathrm{AuD}_{0.036}(\mathbf{2 0 n m}) / \mathrm{PdD}_{0.86}$ & $2.8 \mathrm{E}-14$ & $1.4 \mathrm{E}-14$ & $-\cdots$ \\
\hline $\mathrm{AuD}_{0.036}(\mathbf{7 0 n m}) / \mathbf{P d D}_{0.86}$ & $5.1 \mathrm{E}-15$ & $4.6 \mathrm{E}-15$ & $-\cdots$ \\
\hline
\end{tabular}


The enhancement factor $R_{\mathrm{r}}$ will be compared with the cross section enhancement factor, the ratio of $\sigma(E)$ to that of bare nuclei $\sigma_{\mathrm{b}}(E)$ which is given by eq.(3) with $\Phi\left(r / r_{\mathrm{U}}\right)$ replaced by unity. It is sometimes convenient to discuss the screening effect in terms of an effective screening potential $U_{\mathrm{s}}[4]$, a potential energy inside a negatively charged spherical surface. In this case the cross section $\sigma(E)$ for a given $U_{\mathrm{s}}$ is expressed as;

$$
\sigma(E)=\frac{S\left(E+U_{\mathrm{s}}\right)}{E+U_{\mathrm{s}}} P_{\mathrm{b}}\left(E+U_{\mathrm{s}}\right)
$$

where $P_{\mathrm{b}}$ is the penetration probability for bare nuclei, which is calculated as the exponential factor in eq.(3) with $\Phi\left(r / r_{\mathrm{U}}\right)$ replaced by unity. It is worthwhile to note that the universal potential gives the energy dependence of the cross section enhancement factor very close to that of eq.(5) with $U_{\mathrm{s}}=60 \mathrm{eV}$, as will be shown later in Fig.7.

\section{Results and Discussion}

\subsection{Evolution of $D(d, p) t$ reaction yield}

First of all, it should be mentioned that we were unable to identify any reaction products of the $\mathrm{DD}(\mathrm{d}, \mathrm{np})^{4} \mathrm{He}, \mathrm{DD}(\mathrm{d}, \mathrm{d})^{4} \mathrm{He}$ nor $\mathrm{DD}(\mathrm{d}, \mathrm{t})^{3} \mathrm{He}$ reactions during the experiments done so far with use of $\mathrm{Pd}$ and $\mathrm{Au} / \mathrm{Pd}$. However, the $\mathrm{D}(\mathrm{d}, \mathrm{p}) \mathrm{t}$ reaction has shown anomalous behavior under some conditions. In the following, the $D(d, p) t$ reaction rate is evaluated and discussed in terms of the enhancement factor $R_{\mathrm{r}}$.

Figure 3 shows two examples of temporal behavior of the reaction probability expressed as the enhancement factor during $15-\mathrm{keV} \mathrm{D}_{2}{ }^{+}$irradiation of the Pd sample with no Au layer deposited on the surface. Since the sample has been subjected to repeated use, the zero fluence in the abscissa does not correspond to the beginning of irradiation. The data shown are in the stationary phase when the target was saturated with injected deuterium. Naturally we expect that the molecular ion split into an atom and an ion with the same velocity within several monolayers of the target surface, and therefore that the

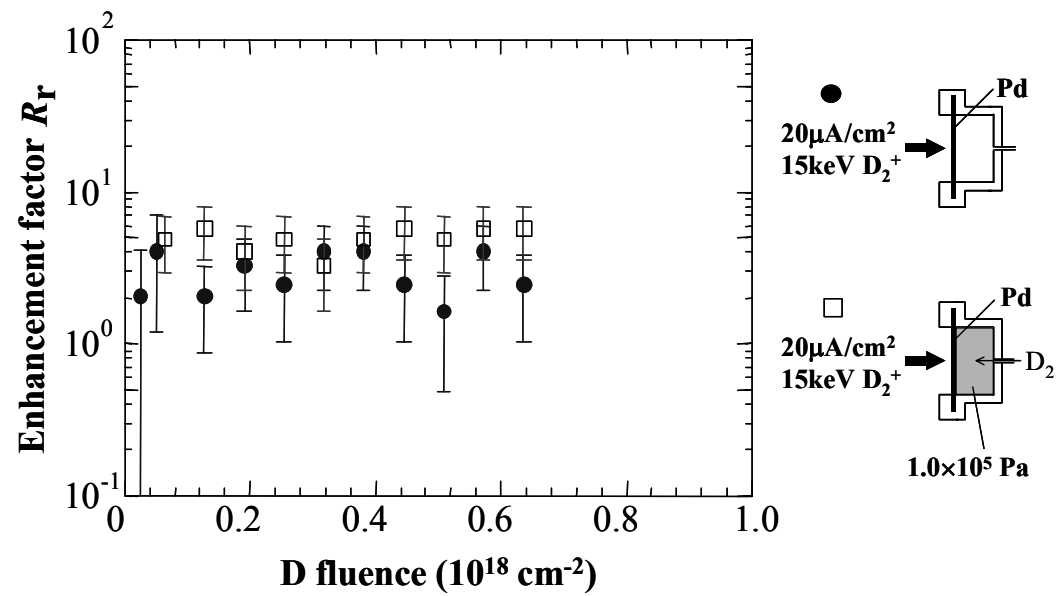

Fig.3. Temporal behavior of the reaction probability expressed as the enhancement factor during $15-\mathrm{keV} \mathrm{D}_{2}^{+}$ irradiation of Pd samples with no Au layer deposited. 
reaction probability is approximated by that for two independent deuterons with the half energy retaining charge equilibrium.

The enhancement factor $R_{\mathrm{r}}$ remains below 6 in both cases. Recalling the uncertainty of a factor of 3 mentioned in the preceding section, we might conclude that the target is saturated with deuterium over the depth region of projectile range and the reaction rate shows no anomaly in the case of the $\mathrm{D}_{2}{ }^{+}$irradiation of Pd with no Au layer deposited.

On the contrary, when the Pd sample was coated with a Au layer, the reaction yield has grown up. Three examples of the evolution of the reaction probability expressed as $R_{\mathrm{r}}$ are shown in Fig.4. In all cases $R_{\mathrm{c}}$ starts at about 10 at the fluence of $1 \times 10^{17} \mathrm{~cm}^{-2}$, and remains well above 10 throughout the run. As the deuteron energy is decreased from $12.5 \mathrm{keV}$ to $7.5 \mathrm{keV}, R_{\mathrm{r}}$ increases up to several hundred.

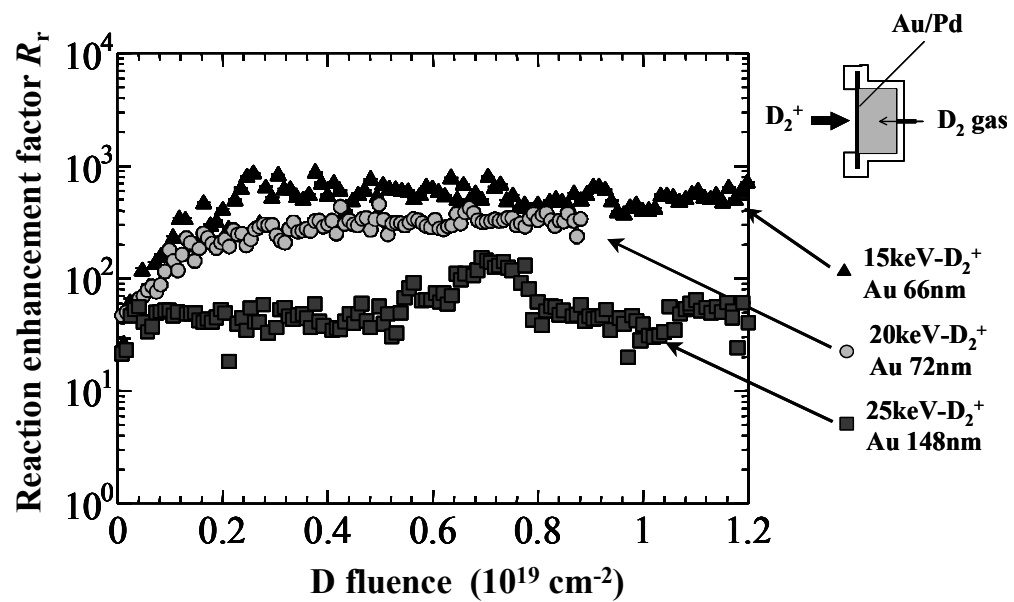

Fig.4. Evolution of the reaction probability expressed as the enhancement factor during 15-, 20-and 25-keV $\mathrm{D}_{2}^{+}$irradiation of the $\mathrm{Au} / \mathrm{Pd}$ samples.

As mentioned in the preceding section, $R_{\mathrm{c}}$ has been calculated for the target with the atomic composition of $\mathrm{PdD}_{0.86}$ to avoid complexity introduced when dynamic change in thickness and composition of the $\mathrm{Au} / \mathrm{Pd}$ layer is taken into account. As can be seen in Table $1, R_{\mathrm{c}}$ would be even greater when the target is assumed to have a deposited layer of $\mathrm{Au}$, and therefore $R_{\mathrm{r}}$ could be an underestimated value by more than one order of magnitude.

In the cases shown in Fig.4, the data are shown from the very beginning of the irradiation of each sample. Another point to be noted is that $R_{\mathrm{c}}$ exceeds 10 even at the very low fluence of $1 \times 10^{17} \mathrm{~cm}^{-2}$. While the number of deuterium atoms existing in a 20$\mathrm{nm}$-thick layer of $\mathrm{PdD}_{0.86}$ is $1.2 \times 10^{17} \mathrm{~cm}^{-2}$, the TRIDYN code predicts that the fluence necessary for the first $20 \mathrm{~nm}$ layer, where most of the reaction events take place, to become saturated is about $2-3 \times 10^{18} \mathrm{~cm}^{-2}$ for a pure sample of $\mathrm{Pd}$. Although the $\mathrm{D}_{2}$ gas filling prior to the irradiation could led the sample composition to the saturation, it is rather difficult to explain the reason for the high $R_{\mathrm{c}}$ at such an early phase of irradiation.

Systematic errors in the experimental results could be caused by contamination of the molecular $\mathrm{D}_{2}{ }^{+}$beams by atomic ions $\mathrm{D}^{+}$and/or neutral $\mathrm{D}$ atoms. In the present system, 
the mass separation of the ions emitted from the ion source is accomplished by the Wien filter. Care should be taken of incidence of neutral particles onto the target. Since most of these neutrals would have the same energy as the molecular ions, even a little amount of the neutrals could make appreciable contribution to the reaction yield. To check this point, dependence of $R_{\mathrm{m}}$ on the ion current density was examined. The current density of the beam was controlled by using downstream optics, i.e., an einzel lens and an electrostatic deflector, since they are expected to change the ion current density with a minimum effect on the neutral current. It has been found that the contribution of neutrals to the proton counting is at most $10 \%$ and that the contribution of target events to the neutron counting is about $40 \%$. Moreover, it was confirmed by examining the charge-tomass ratio spectra of the beam coming out from the Wien filter that $\mathrm{D}_{2}^{+}$and $\mathrm{D}^{+}$ions are well separated from each other so that $\mathrm{D}^{+}$ions have very little chance to make appreciable contribution to $R_{\mathrm{m}}$.

Finally it should be stressed that such large values of $R_{\mathrm{r}}$ has never been observed in experiments using $\mathrm{TiD}_{\mathrm{x}}$ as the sample target [12].

\subsection{Change in atomic composition}

The samples have been analyzed by means of RBS and ERDA methods using a 4-MeV ${ }^{4} \mathrm{He}$ beam. The typical energy spectra of RBS made under $\mathrm{D}_{2}^{+}$ irradiation at the $\mathrm{D}$ fluence of zero, $4.5 \times 10^{18} \mathrm{~cm}^{-2}$ and $1.1 \times 10^{19}$ $\mathrm{cm}^{-2}$ are shown in Fig.5. The continuum spectra below 3.1 $\mathrm{MeV}$ correspond to the $\operatorname{Pd}(\alpha, \alpha) \operatorname{Pd}$ scattering, and the spectra with the peak near 3.4 $\mathrm{MeV}$ correspond to the $\operatorname{Au}(\alpha, \alpha)$ Au scattering. With the progress of the $\mathrm{D}_{2}{ }^{+}$irradiation, the $\mathrm{Au}(\alpha, \alpha) \mathrm{Au}$ scattering yield decreases and its spectral shape is also deformed. In addition, the

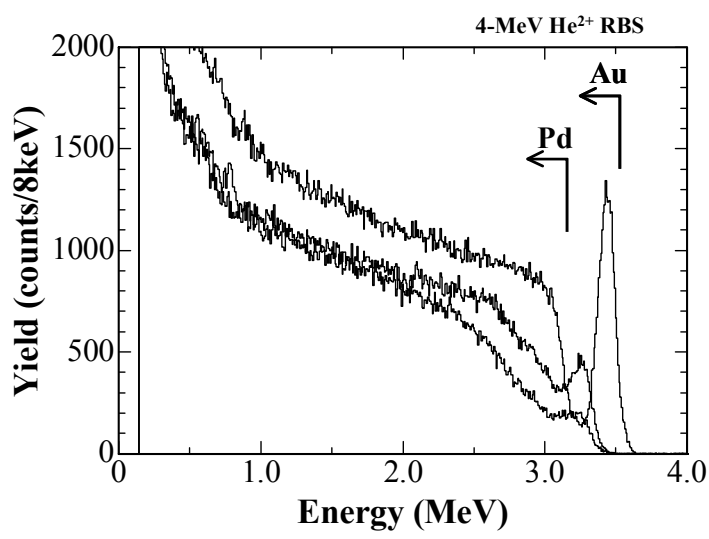

Fig.5. Energy spectra of RBS taken at the fluence of zero (upper), $4.5 \times 10^{18} \mathrm{~cm}^{-2}$ (middle) and $1.1 \times 10^{19} \mathrm{~cm}^{-2}$ (lower) during $\mathrm{D}_{2}{ }^{+}$irradiation of a $\mathrm{Au}(70 \mathrm{~nm}) / \mathrm{Pd}$ sample. The bottoms of the arrows represent the detection energy of ${ }^{4} \mathrm{He}$ scattered by $\mathrm{Au}$ and $\mathrm{Pd}$ atoms on the sample surface, respectively, while the horizontal length of the arrows corresponds the target depth of $300 \mathrm{~nm}$.

slope of the spectral edge formed by the $\operatorname{Pd}(\alpha, \alpha) \operatorname{Pd}$ scattering becomes smaller.

The decrease in the $\mathrm{Au}(\alpha, \alpha) \mathrm{Au}$ scattering yield can be caused both by the sputtering loss of the Au atoms and by formation of a mixed layer of Au and Pd. The peak flattening and the edge moderation, however, can be explained only by mixing of $\mathrm{Au}, \mathrm{Pd}$ and possible other elements in a depth region of the order of several hundred $\mathrm{nm}$.

Naturally, TRIDYN predicts the mixing of atoms in the target, but with less violently; the FWHM of the mixed layer is about $60 \mathrm{~nm}$ at the fluence of $1 \times 10^{19} \mathrm{~cm}^{-2}$ and 
the region of Pd saturated with deuterium reaches $300 \mathrm{~nm}$. The latter is compared with the experimental results in the following.

\subsection{Evolution of deuterium density}

The deuterium density profiles are shown in Fig.6-(a) and -(b) for the case of $\mathrm{D}_{2}{ }^{+}$ irradiation of the $\mathrm{Au}(70 \mathrm{~nm}) / \mathrm{Pd}$ sample during irradiation with $15 \mathrm{keV}$ and $20 \mathrm{keV} \mathrm{D}_{2}{ }^{+}$, respectively. These have been reconstructed from the energy spectra of recoil particles without any correction for overlapping of recoil hydrogen having smaller kinematic factor, i.e., smaller energy. Therefore, in the depth region deeper than several hundred $\mathrm{nm}$, the deuterium depth profiles are contaminated by the hydrogen profile with improper depth scale. The resolution of the method in the present configuration is about $100 \mathrm{~nm}$.
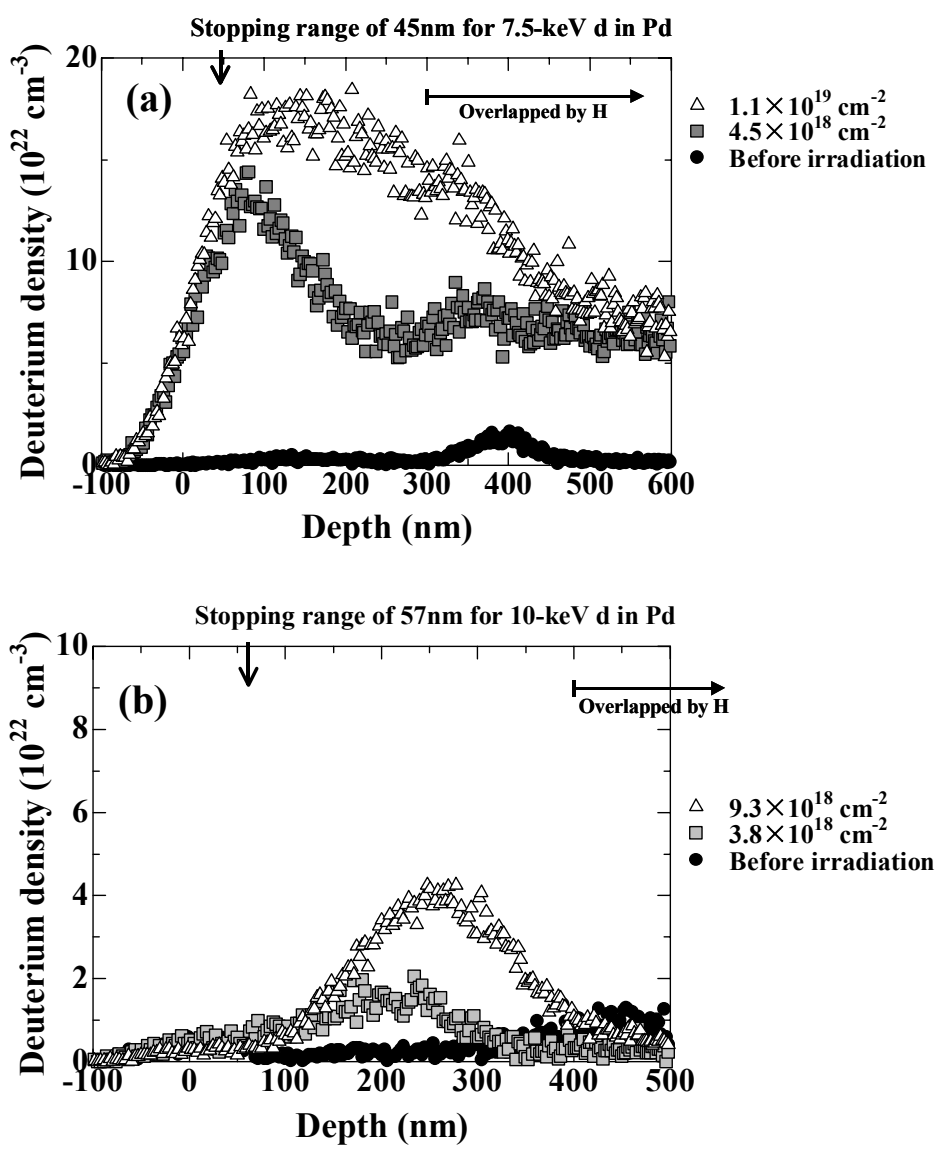

Fig.6. Evolution of the depth profile of deuterium density in the $\mathrm{Au}(70 \mathrm{~nm}) / \mathrm{Pd}$ sample during irradiation with $15 \mathrm{keV}$ (a) and $20 \mathrm{keV} \mathrm{D}_{2}{ }^{+}(\mathrm{b})$.

The deuterium density increases with increasing irradiation fluence. In the case of (a) the maximum deuterium density of $1.8 \times 10^{23} \mathrm{~cm}^{-3}$ reached at the fluence of $1.1 \times 10^{19}$ $\mathrm{cm}^{-2}$ corresponds to a composition of $\mathrm{PdD}_{2.7}$. This is extraordinarily higher than the 
saturated deuterium density of $5.8 \times 10^{22} \mathrm{~cm}^{-3}$ corresponding to $\mathrm{PdD}_{0.86}$. However, the extraordinarily high density has not been reproduced in other cases including that of Fig.6-(b).

The most interesting fact is that the depth at which the deuterium density has the maximum is far beyond the stopping range of the projectile of several tens of $\mathrm{nm}$. It is in the mixed layer of $\mathrm{Au}$ and Pd or around the boundary of the mixed layer and the bulk Pd. It is rather surprising that the deuterium density in the surface region, where the projectile is supposed to have relatively large probability of the $\mathrm{D}(\mathrm{d}, \mathrm{p}) \mathrm{t}$ reaction, is rather low compared to that in the deeper region. When we take this fact into account, the enhancement factor $R_{\mathrm{r}}$ would be of the order of $10^{4}$ or even greater.

We infer that some mechanisms to enhance the reaction rate or the reaction cross section are working in this mixed layer of Au and Pd loaded with high density of deuterium. The lattice defects or the lattice composed of Au and Pd could trap more deuterium than the interstitial sites of Pd, which could lead to high density of deuterium and induce the $\mathrm{D}(\mathrm{d}, \mathrm{p}) \mathrm{t}$ reaction with extraordinary high rate.

\subsection{Dependence of $\boldsymbol{R}_{\mathrm{r}}$ on the projectile energy and range}

The enhancement factor $R_{\mathrm{r}}$ obtained so far are summarized in Fig. 7 as a function of energy. Here the energy is expressed as the deuteron energy in the center of mass system of the projectile deuteron and the target deuterium at rest to compare with the cross section enhancement factor mentioned earlier. We see here that in most cases of $\mathrm{D}_{2}{ }^{+}$or $\mathrm{D}_{3}{ }^{+}$ irradiation significant enhancement greater than 100 has been achieved. On the other hand, $\mathrm{D}^{+}$irradiation causes little effect on the reaction enhancement. We have expected that the enhancement factor is a decreasing function of energy. It is, however, rather difficult to find a unique line of cross section enhancement for a unique shielding potential fitted to the experimental data.

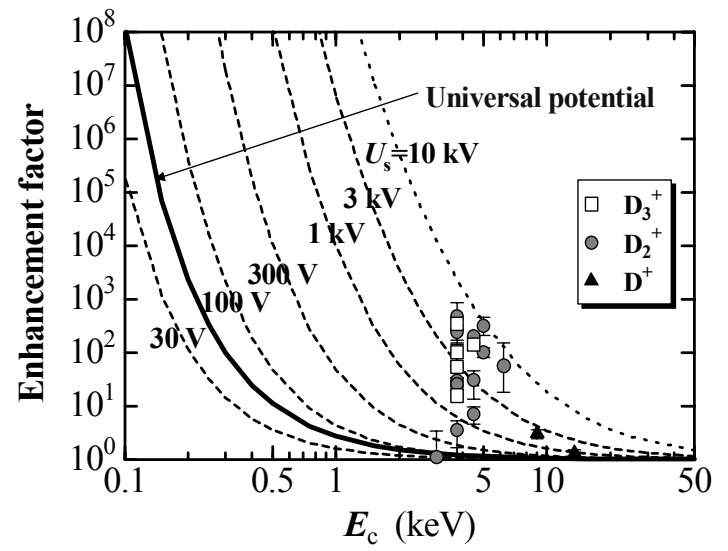

Fig.7. Enhancement factor compared with the cross section enhancement factor as a function of energy in the center-ofmass system of the projectile and the target deuterium at rest.

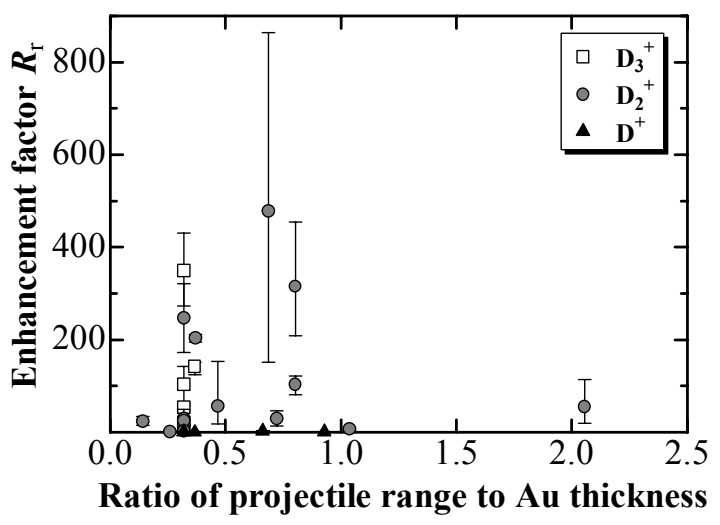

Fig.8. Enhancement factor as a function of the ratio of the projectile range to the initial thickness of the Au layer. 
The same data are plotted in Fig. 8 as a function of the ratio of the projectile range to the thickness of the Au layer. In view of the deuterium density profiles, we expected some dependence of $R_{\mathrm{r}}$ on the projectile range. However, we find no apparent dependence on the range.

There might be another factor governing the reaction enhancing mechanism, or the experimental condition to realize the enhanced reaction might be very sensitive to a factor we have not yet controlled. We need further experimental evidence to confirm the anomaly.

Anyway it should be again noted that $R_{\mathrm{r}}$ would be even higher, when we take account of the deuterium density not reaching the saturation value in the surface region where most of the conventional beam-target reactions take place.

\section{Summary}

The reaction enhancement factor $R_{\mathrm{r}}$ reaching the maximum value of the order of $10^{3}$ has been recorded under the $15-25 \mathrm{keV} \mathrm{D}_{2}{ }^{+}$irradiation of Au-deposited Pd samples which has formed the mixed layer of $\mathrm{Au}$ and $\mathrm{Pd}$ in the near-surface region. The deuterium density distributions have been observed to have their maxima at substantially deeper location than the projectile range and sometimes higher than the saturation density of $\mathrm{PdD}_{0.86}$. We speculate that the formation of the $\mathrm{Au} / \mathrm{Pd}$ mixed layer retaining the high deuterium density induces the enhancement of the reaction rate.

\section{References}

[1] J. Kasagi, T. Ohtsuki, K. Ishii and M. Hiraga. J. Phys. Society Japan. 64 (1995) 777783.

[2] A. Takahashi, K. Murata, K. Ochiai, H. Miyamaru and T. Iida. Fusion Technol. 34 (1998) 256-270.

[3] A. Takahashi, K. Murata, K. Ochiai, H. Miyamaru. Phys. Letters A. 255 (1999) 8997.

[4] J. Kasagi, H. Yuki, T. Baba, T. Noda, T. Ohtsuki and A. G. Lipson. J. Phys. Soc. Jpn. 71 (2002) 2881-2885.

[5] Y. Iwamura, M. Sakano and T. Itoh. Jpn. J. Appl. Phys. 41 (2002) 4642-4650.

[6] A. G. Lipson, et al.; Proc. ICCF9 (Tsinghua Univ., 2003) 213.

[7] A. Kitamura, T. Saitoh and H. Itoh. Fusion Technol. 29 (1996) 372.

[8] M. Miyamoto, Y. Awa, N. Kubota, A. Taniike, Y. Furuyama and A. Kitamura. Proc. 9th Int. Conf. on Cold Fusion, Beijing, China (Tsinghua Univ. Press, 2003) 261-264.

[9] J. F. Ziegler, J. P. Biersack and U. Littmark. The Stopping and Range of Ions in Solids. (Pergamon Press, 1985).

[10] W. Eckstein. Computer Simulation of Ion-Solid Interactions. (Springer-Verlag, 1991)

[11] A. Krauss, H. W. Becker, H. P. Trautvetter and C. Rolfs. Nucl. Phys. A465 (1987) 150 . 
[12] N. Kubota, A. Taniike and A. Kitamura. Conference Proceedings Vol.70 "ICCF8" (F. Scaramuzzi (Ed.), Soc. Italiana di Fisica, 2000) 311. 